better, and metric radiographic joint space width (rJSW) is related to subsequent risk of radiographic progression. Although new MRI measures provide increased responsiveness in DMOAD trials, it is unknown whether selecting for recruitment based on radiographic criteria are well suited for responsiveness of these new measures.

Objectives: (1) To determine which baseline rJSW values are associated with most subsequent progression for rJSW, MRI cartilage and bone outcomes (2) Explore baseline covariates that influence progression rates (3) Estimate the trial numbers needed using the criteria determined by steps (1) and (2).

Methods: We used all knees from the Osteoarthritis Initiative which had all 3 measures recorded (rJSW - Duryea method; MRI cartilage thickness \& bone area, Imorphics) at baseline, 1 and 2 years. We categorised knees into bins of $1 \mathrm{~mm}$ rJSW, and assessed the 2 year changes of each bin, and characterised the distribution of rJSW in KL 0 knees. We used ANCOVA models to consider which covariates (including gender, height, weight, alignment, age, pain severity) affected 2-year slope of change, and responsiveness using SRM. For the final optimised recruitment groups, we calculated SRMs (Cls assessed using the bootstrap method of Efron) and derived the number of patients per arm in a putative trial.

Results: 4796 knees were included (2789 females, mean age 61.45). The lower 95th percentile values for rJSW in women and men were 3.9 and $4.5 \mathrm{~mm}$ respectively. The mean changes at 2-years for all 3 outcomes were greatest for the categories of $2-3$ and $3-4 \mathrm{~mm}$ baseline rJSW (Figure $1 \mathrm{~A}$ ) with notably little change in knees with rJSW $<2 \mathrm{~mm}$. Of the covariates, only pain improved responsiveness. Using a total WOMAC pain criterion; $\geq 3 / 20$ reduced numbers from 726 knees to 331 knees, and improved 1 year SRM $(95 \% \mathrm{Cl}$ ) from 0.27 $(0.17,0.34)$ to $0.41(0.29,0.51)$ in rJSW, from $0.45(0.37,0.51)$ to $0.55(0.45,0.65)$ in MRI cartilage and from $0.60(0.52,0.66)$ to $0.73(0.62,0.83)$ in MRI bone. Figure 1B shows the relative SRMs for the 3 outcomes based on 2 inclusion criteria (rJSW $2-4 m m$ and pain $>3 / 20 ; n=331$ ) and demonstrates the required trial numbers (with confidence intervals) based on the SRMs.
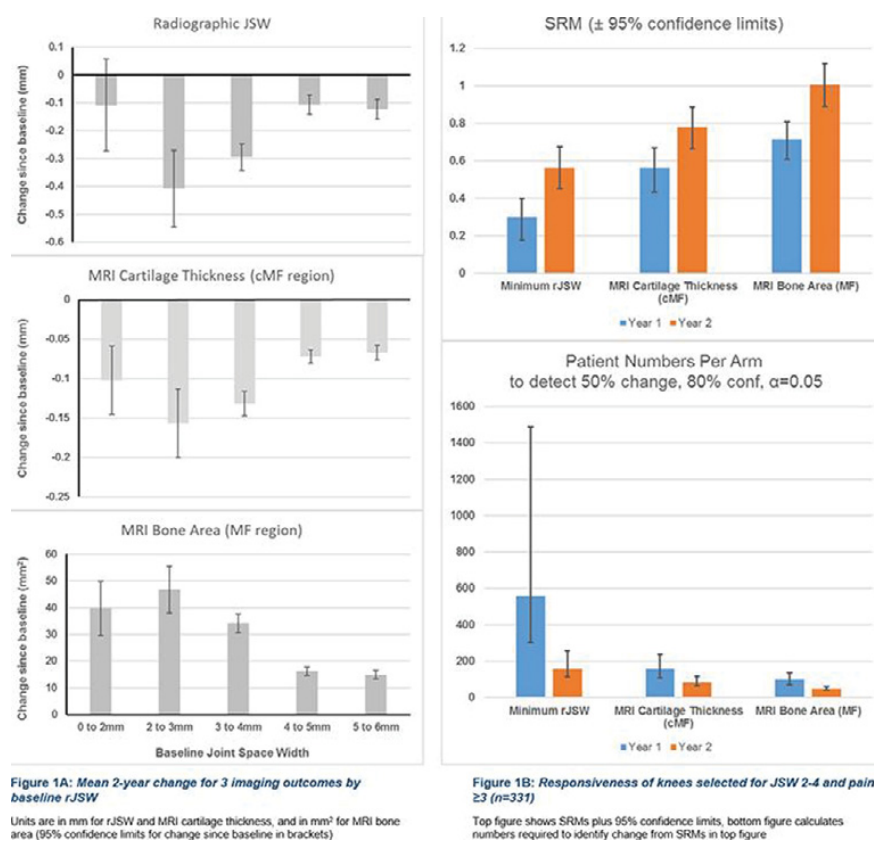

Conclusions: Selecting patients based on 2 simple criteria will improve responsiveness in clinical trials for all 3 imaging outcomes using standard imaging outcomes. Selecting for rJSW of $2-4 \mathrm{~mm}$ is most important while adding a pain criteria further improves responsiveness; no other covariates improved this. Caution should be applied when using SRM to power a study because of the inherent difficulties in calculating standard deviations; Fig 1B shows for example that the confidence limits for rJSW at 12 months vary from 302 to 1489 . This analysis also confirms the advantages of MRI outcomes over rJSW in terms of study size and duration: a 12-month study with cartilage thickness or bone area endpoints needs no more than 238 or 137 patients (including the upper 95th percentile confidence limit).

Disclosure of Interest: M. Bowes Employee of: Imorphics Ltd, G. Guillard Employee of: Imorphics Ltd, A. Brett Employee of: Imorphics Ltd, G. Vincent Employee of: Imorphics Ltd, P. Conaghan: None declared

DOI: 10.1136/annrheumdis-2017-eular.6860

\section{OP0165 A NEOEPITOPE FRAGMENT OF C-REACTIVE PROTEIN IS PROGNOSTIC OF RADIOGRAPHIC KNEE OA}

A.C. Bay-Jensen ${ }^{1}$, A. Bihlet ${ }^{2}$, I. Byrjalsen ${ }^{2}$, J.R. Andersen ${ }^{2}, \mathrm{Y}^{2} \mathrm{He}^{1}$, A.S. Siebuhr ${ }^{1}$, C.S. Thudium ${ }^{1}$, B.J. Riis ${ }^{2}$, C.H. Christiansen ${ }^{2}$, M.A. Karsdal ${ }^{1}$. ${ }^{1}$ Rheumatology, Nordic Bioscience Biomarkers and Research; ${ }^{2}$ Clinical development, Nordic Bioscience, Herlev, Denmark

Background: There are two major needs in clinical development of DMOADs: 1) Identifying a suitable population with an active and progressive disease in order to demonstrate significant improvements by an efficacious intervention; and 2) Phenotyping patients and linking them to a corresponding treatment mode-ofaction (e.g. anti-inflammatory). The biochemical marker CRPM is a neoepitope of $\mathrm{C}$-reactive protein (CRP) and is released from the local inflamed tissue when CRP is degraded by proteases such as matrix metalloproteinases

Objectives: The purpose of this study was to translate the CRPM, from rheumatoid arthritis (RA), where it has been extensively tested, to OA, and to test whether it is predictive of radiographic $O A$

Methods: The placebo arms of two phase III OA trial (clinicaltrial.gov: NCT00486434 and NCT00704847), a phase III RA study (the LITHE study, $\mathrm{N}=490$ ), which included patients with active, moderate-severe RA (NCT00106535), as well as in an early $\mathrm{RA}$ cohort $(\mathrm{N}=92)$ were used. Subjects with symptomatic and radiographic knee OA: WOMAC pain $\geq 150 \mathrm{~mm}$ and/or WOMAC function $\geq 510 \mathrm{~mm}$, and Kellgren-Lawrence grade $(\mathrm{KLG}) 2$ or 3 . KLG were scored for both knees at baseline and year 2 (Y2). Serum CRPM and hsCRP were measured at baseline. The association between serum CRPM levels and disease activity score (DAS28) and hsCRP was investigated by Spearman's correlations. Quartile ranges of CRPM in the early RA cohort were used to define the cut-off between inflammatory $\mathrm{OA}$ and non-inflammatory $\mathrm{OA}$. OA knees were divided into cases and controls based on a terminology proposed by the FNIH-OAI consortium (1); knees with $K L G \geq 2$ at $B L$ were excluded, and incidence $O A$ at $Y 2$ was defined $K L G \geq 2$. Logistic regression was used to compare cases and controls.

Results: There was a significant correlation between disease activity measures and CRPM in both RA studies. Seventy-five percent of the LITHE patients had high or very high levels of CRPM at BL, which was changed to a pattern similar to early RA after treatment. The mean CRPM levels were significantly lower in $\mathrm{OA}(8.5[95 \% \mathrm{Cl} 8.3-8.8] \mathrm{ng} / \mathrm{mL})$ compared to the RA patients (15.6 [9.5-21.6] $\mathrm{ng} / \mathrm{mL})$; however, a significant subset of OA patients (41\% and $31 \%$ in SMC2301 and SMC2302) had CRPM levels $>9 \mathrm{ng} / \mathrm{mL}$, as $75 \%$ of patients with early RA. Patients with $\mathrm{BL}$ or $\mathrm{Y} 2 \mathrm{CRPM}$ levels $\geq 9 \mathrm{ng} / \mathrm{mL}$ were more likely to develop knee $\mathrm{OA}$ than patients with low level of CRPM. Overall, moderate to very high levels of CRPM at BL and Y2 were predictive of incidence OA with odds ratio of 4.6 [1.2-17] and 2.5 [1.2-4.8].

\begin{tabular}{|c|c|c|c|c|c|c|}
\hline \multicolumn{2}{|l|}{ Table } & \multirow{2}{*}{$\begin{array}{l}\text { Early RA } \\
0.54(<0.0001)\end{array}$} & \multicolumn{2}{|c|}{ Moderate-Severe RA } & \multirow[t]{2}{*}{ SMC2301 } & \multirow[t]{2}{*}{ SMC2302 } \\
\hline hsCRP & Rho (p-value) & & $0.66(<0.0001)$ & & & \\
\hline DAS28 & Rho (p-value) & $0.43(<0.0001)$ & $0.24(<0.0001)$ & & & \\
\hline \multirow{2}{*}{\multicolumn{2}{|c|}{ CRPM level }} & Quartile range & \multicolumn{4}{|c|}{ No. of patients (\%) } \\
\hline & & & Before treatment & After treatment & $n_{1}$ & $n_{2}$ \\
\hline \multicolumn{2}{|l|}{ Low } & $<9 \mathrm{ng} / \mathrm{mL}$ & $49(8.2 \%)$ & $87(18 \%)$ & $267(59 \%)$ & $233(69 \%)$ \\
\hline \multicolumn{2}{|c|}{ Moderate } & $9-12$ & $100(17 \%)$ & $123(25 \%)$ & $137(31 \%)$ & $71(21 \%)$ \\
\hline \multicolumn{2}{|l|}{ High } & $12-15$ & $132(22 \%)$ & $105(21 \%)$ & $26(6 \%)$ & $23(7 \%)$ \\
\hline \multicolumn{2}{|c|}{ Very high } & $>=15$ & $318(53 \%)$ & $175(36 \%)$ & $19(4 \%)$ & $11(3 \%)$ \\
\hline
\end{tabular}

Conclusions: A subset of OA patients, up to $41 \%$, appear to have tissue inflammation comparable to that of RA, reflected by the level of CRPM. Furthermore, high CRPM levels was prognostic of incident knee OA. These data suggest that CRPM is blood-based biochemical marker for finding OA patients with an inflammatory phenotype.

References:

[1] Longitudinal validation of periarticular bone area and 3D shape as biomarkers for knee OA progression? Data from the FNIH OA Biomarkers Consortium.Hunter D et al.Ann Rheum Dis. 2016 Sep;75(9):1607-14.

Disclosure of Interest: A. Bay-Jensen Shareholder of: Nordic Bioscience, Grant/research support from: DBOARD, Employee of: Nordic Bioscience, A. Bihlet Shareholder of: Nordic Bioscience, Employee of: Nordic Bioscience, I. Byrjalsen Employee of: Nordic Bioscience, J. Andersen Shareholder of: Nordic Bioscience, Employee of: Nordic Bioscience, Y. He Employee of: Nordic Bioscience, A. S. Siebuhr Employee of: Nordic Bioscience, C. Thudium Employee of: Nordic Bioscience, B. Riis Shareholder of: Nordic Bioscience, C. Christiansen Shareholder of: Nordic Bioscience, M. Karsdal Shareholder of: Nordic Bioscience, Employee of: Nordic Bioscience

DOI: 10.1136/annrheumdis-2017-eular.6290 